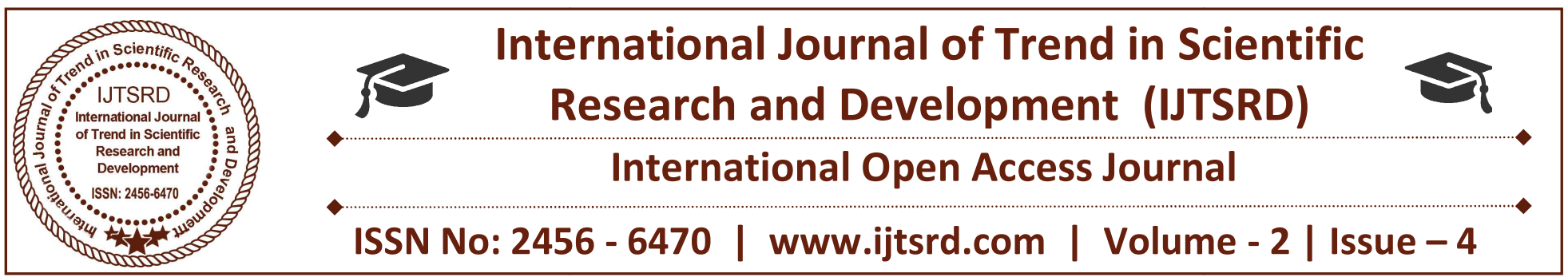

\title{
Impacts of Big Data on Smart Farming
}

\author{
Akshatha S Kumar ${ }^{1}$, Deepthi S Kumar ${ }^{2}$, Manjunath $\mathbf{C ~ R}^{3}$, Soumya K N \\ ${ }^{1,2}$ B.Tech Final Year, ${ }^{3}$ Associate Professor, ${ }^{4}$ Assistant Professor \\ ${ }^{1,2,4}$ Department of Information Science \& Engineering, ${ }^{3}$ Department of Computer Science \& Engineering, \\ ${ }^{1,2,3,4}$ School of Engineering \& Technology - Jain University, Bangalore, Karnataka, India
}

Abstract-- Farming is the broadest monetary segment and assumes as a critical part in general financial advancement of a country. Technological headway in this field of agribusiness will find a way to build the cultivating exercises. Smart farming has a potential to deliver a productive and sustainable agricultural production, based on a precise and resource efficient approach. Smart farming is an improvement that underscores the utilization of data and correspondence innovation in the digital physical farm administration cycle. New advancements for example the internet of things and cloud computing are relied upon to use this improvement and present more robots and computerized reasoning in cultivation. This is done by the big data gigantic volumes of information with a wide assortment that can be caught, broken down and utilized for basic leadership. This survey expects to pick up understanding into the nest in class of Big data applications in smart farming and distinguish the financial difficulties to be tended to.

Keywords - Smart Farming, Big Data in Smart Farming

\section{INTRODUCTION}

Big data analytics is the technique of examining substantial and varied data sets i.e., big data -- to discover covered patterns, unexplained correlations, advertise trends, consumer personal tastes and more profitable information which can assist organizations make moreinformed business decisions. Big data analytics has acquired in numerous domains and one such is agriculture, predominantly. Big data is predicted to possess a huge impact on smart farming and involves the entire supply chain. It incorporates some smart sensors and gadgets that deliver enormous measures of information that give phenomenal basic leadership capacities. Smart Farming is one such development that stresses the utilization of Information and Communication technology in the cyber-physical farm management cycle. New advancements, such as, the Internet of Things and Cloud Computing are required to use this improvement and present more robots and computerized reasoning in cultivating. The extent of Big Data applications in Smart Farming goes beyond primary production; it is impacting the whole nourishment production network. Big data is being utilized to give prescient bits of knowledge in cultivating activities, drive ongoing operational choices, and overhaul business. The interest for big data in smart farming is extremely high as a result of its stockpiling (high volume) and computational power. The eventual fate of Smart Farming may unwind in a continuum of two outrageous situations:

1. Closed, restrictive frameworks in which the farmer is a piece of an exceedingly coordinated sustenance production network.

2. Open, collective frameworks in which the farmer and each other partner in the chain arrange are adaptable in picking business accomplices too for the innovation concerning the food production side. Big Data technologies are playing a basic, complementary part in this improvement: machines are furnished with all sorts of sensors that measure information in their condition that is utilized for the machines' conduct.

\section{BACKGROUND WORK}

A smart farm sensing framework and mobile smart irrigator that proceeds on mechanical extension slider course of action. Both the frameworks comprise of microcontrollers, sensors, and the GSM module to 
connected with each other and with the outer condition. The smart sensing framework detects the dampness content with the guide of the soil moisture sensor. The deliberate information from the smart sensing framework is sent to the smart irrigator through the GSM module. The agriculturist can have control over the framework by having a remote correspondence with GSM module through his cell phone. Sensor utilized here is INFRARED SENSOR: The producer is an IR LED (light emitting diode) and the detector is an IR photodiode, which is delicate to IR light of an indistinguishable wavelength from that discharged by the IR LED. An opto-isolator contains a light source, which is mostly a LED that converts electrical flag into light, a closed optical channel and a photo sensor, which identifies approaching light and either, creates electric vitality specifically or regulates electric current spilling out of an outer power supply. After an appropriate connection has been set up between the two frameworks through the GSM module, the engine introduce in the overhead crane has begun. This engine helps in flat and vertical developments of the Smart Irrigator over the field. In view of the activation of a specific sensor said over, a particular piece of the framework segments like green fertilizer, manures, seeds, and water holder gets enacted to achieve a particular assignment. The smart irrigator framework disposes the troubles faced in manual cultivation. This smart sensing framework provides accurate results and the smart irrigator system manages to spray necessary nutrients according to the requirement of crops. The smart irrigator framework disposes the troubles faced in manual cultivation.

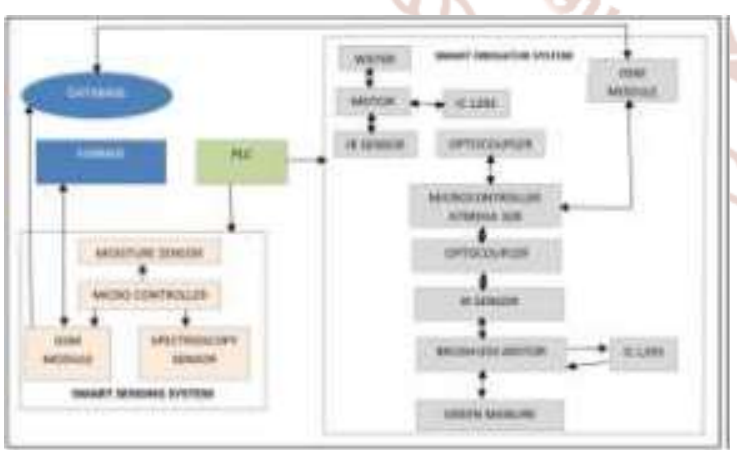

Figure 1: Smart sensing system

The Smart Farming services at the preproduction stage are important in that they support agricultural producers' or wholesalers' choices to maintain a strategic distance from overproduction or lack that may cause money related troubles. These services give related data and consulting when they intend to create or buy before the production begins.

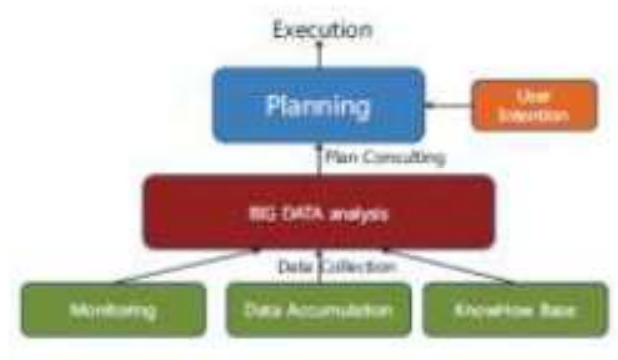

Figure 2: Pre-Production stages

Monitoring function assembles the current natural information from different sensors estimating temperature, moistness, $\mathrm{pH}$, and so on. Current natural information may incorporate development asset data, for example, accessible rural machines, work constrain, and so forth. Information Accumulation Function assembles all related data, for example, the past development records, last benefits per assortments of harvests, and so forth. Knowhow base management function accumulates encounterbased data from clients or client groups. These data from sensors, clients or client groups can be passed on through the media transmission frameworks given by the suppliers. All these accumulated data are exchanged to Data Analysis Function which investigates the assembled data and produces important outcomes that will help the consultation process. The dissected outcomes are exchanged to Plan Consulting Function that connects with services clients, Agricultural Producer or Distributor, to help the clients make decisions.

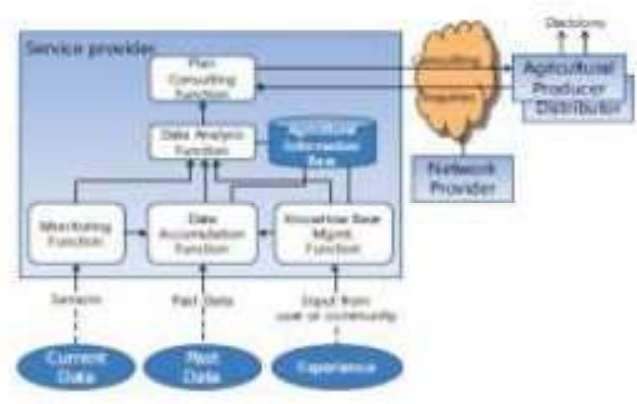

Figure 3: Big data analysis

To beat the issues in the current framework, they have proposed a framework that decreases middle men issues and agriculturists can take in new approaches from the specialists through this framework. In this framework, they can likewise get ready for planting the best product with the assistance of climate alarms that gives data about the climatic conditions and which crop is suitable for the specific atmosphere. This framework gives a stage to the farmers where 
they can know the costs of various markets and offer their product at the best cost.

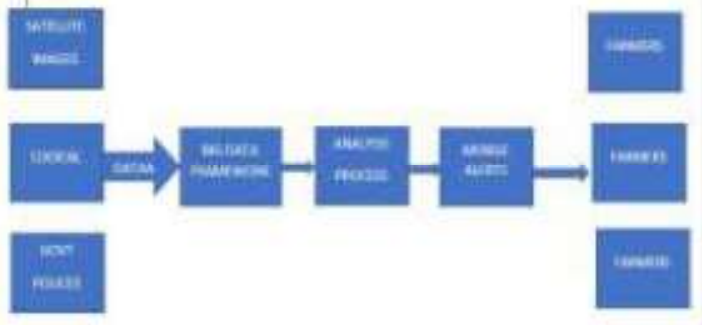

Figure 4: Big data framework

Highlights of the proposed framework are news feed, web-based learning for the farmers and climate alert, purchase items, offer items, advertise status and inquiries. This methodology incorporates diverse stages like data gathering from various sources, data analysis module is a preparing stage which is induced utilizing HIVE, ready framework and representation.

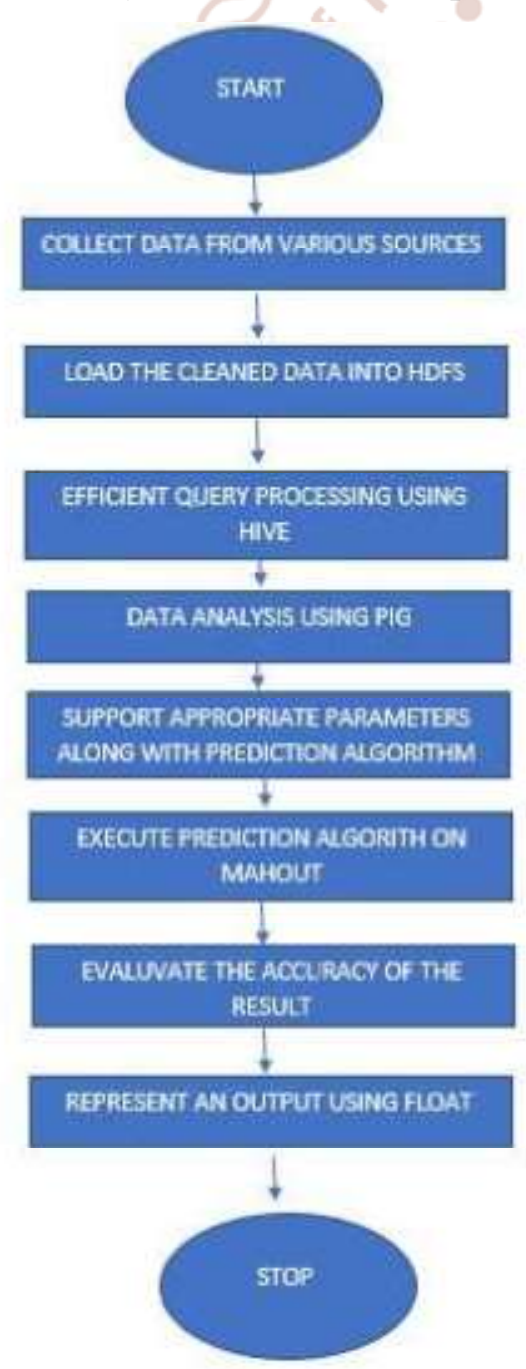

Figure 5: Big data framework algorithm
2.4 This framework comprises of an arduino board which controls distinctive sensors, for example, soil, temperature, dampness, mugginess, light and $\mathrm{pH}$. The soil dampness sensor is set at the most profound purpose of soil with the deferral of 12 hours. Once the soil is identified to be dry the water pump is enacted. The temperature and humidity sensors is set at the focal point of the framework to distinguish the temperature and dampness and customized with a delay of 12 hours 10 minutes to get activated once the soil dampness is low. At the point when the three sensors are detected to be low in the framework, the temperature and humidity sensor are expanded by passing steam which is created by means of a boiler. The light sensors are actuated after the light force nearly moves towards becoming nil. These lights act as a pest absorbent as the pests are attracted towards the light and furthermore give methods for brightening. The sort of harvests and essential parameters are put away in the database and the sensors communicate with the server intermittently. The sensors are treated as nodes, if any node does not react to the server inside a timeout period; the node will be perceived as a defective one. At that point the warning of the defected node is educated by the server which is either supplanted or the blame is rectified.

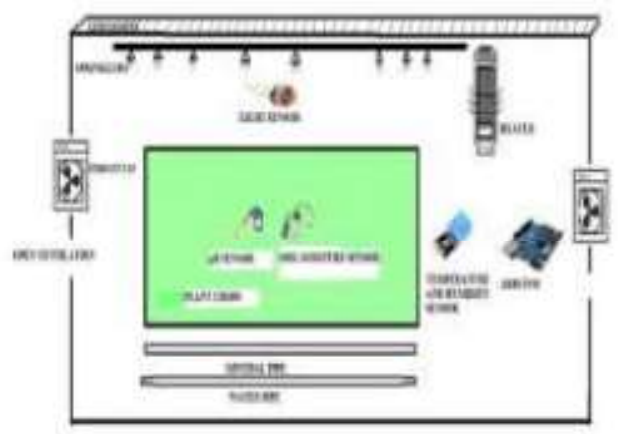

Figure 6: Poly house system

In this paper they have used sensors which collect the information related to crop monitoring and send it to the server. The server will implement actions such as watering plants etc. Then architecture is introduced which is cloud service-oriented architecture which includes cloud services provided for the farmers. They are: Service using web: It encourages a solitary window solution for the farmers and their group. Service using voice: It is the transmission of voice discussions over Internet. It depends on the Voice over Internet Protocol (VoIP). The ranchers can get to the data service from anywhere and anytime with the cell phones. So, the voice benefit is at present a key channel to get connected with the ranchers. The 
ranchers can utilize this service and get information. Service using text messages: This is straightforward, simple and less expensive service for the agriculturist's group to profit the service from out framework. SMS stands for Short Message Service. It is helpful service for sending short messages which works over remote portable system. With the expanding number of ranchers that own cell phones, the data scattering through SMS has turned into a key service. Service based on interactive video conferencing: The most striking highlights of this model lie in a visual and face to face interaction. Ranchers can acquire this service remotely. This is exceptionally valuable service for the agriculturists. They can watch the recordings which are recorded related agribusiness, for example, how to utilize present day farming machinery and can utilize this for their training
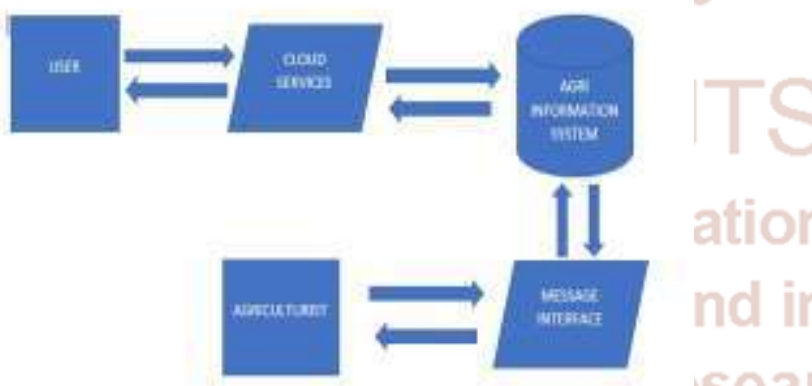

Figure 7: Cloud service-oriented architecture

The system which is proposed here has the following features they are, the information regarding crops and field data is automatically collected as soon as the sensors are fixed and gets stored in the server, the reading of the field, soil and moisture are collected, as soon as the data is collected, the data is backed up and is made sure that the data is secure, the analysis for crops is done

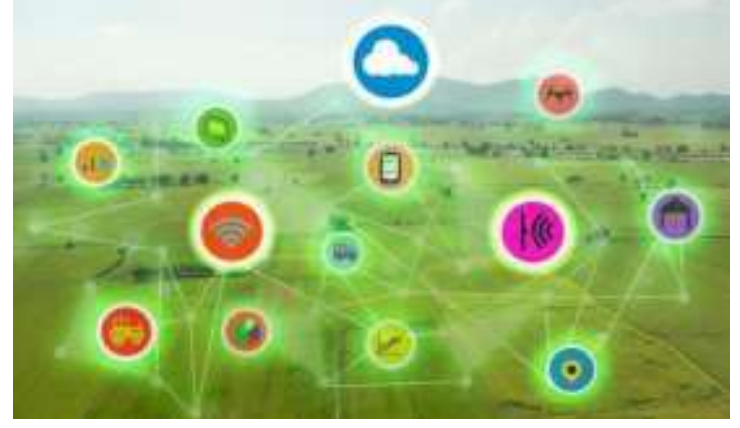

Figure 8: Smart farming

\section{IMPACTS OF BIG DATA}

Agriculture plays a vital role and is the backbone of a country's economic activity. The traditional methods in agricultural practices are becoming grossly incompetent to cater the escalating needs. Thus it has become important to follow unique technologies to enhance agricultural standards. There is in need of a new and effective technology which would improve the productivity, profitability and sustainability of the farming systems. Over a period of time, people tried numerous methodologies in the agricultural field using various technologies. In this technological era, various modern agricultural methods are rapidly increasing. In order to fulfil the needs of the growing population it has become necessary to acquire newer techniques. One such is Big Data Analytics. Many scholars have invented new techniques considering the necessary parameters such as the challenges faced advantages and disadvantages, their impact and so on. Smart farming is one such achievement in the field of big data. Big data has influenced smart farming using various methodologies. The impact of big data on smart farming is discussed below. There are many problems faced in the agricultural areas, one such is that the lack of modern machines included in the agricultural activities. In India there is still use of conventional tools such as plough, sickle and so on which requires a lot of manual work. The smart farming system implemented here will decrease the manual work and helps in agricultural activities. The usage of synthetic fertilizers and pesticides pollutes the ground water but, in this system, it is replaced by the organic fertilizers so that the soil structure is improved. The farmers faced many problems such as electricity shortage,manual labour work, lack of modern machines and lack of knowledge about farming and to use mineral contents. The advantages of this system are solar panels were fixed due to electricity shortage and usage of organic fertilizers instead of macro nutrients. Huge research had to be done to implement this system and all the crop details were included in the database to provide details for the farmers. As observed the system does not look much user friendly and this model can be efficient and may serve its purpose with all the necessary components. An important stage which helps the farmers by giving them useful information and helps them to plan for producing o purchasing products before the production starts which is known as the pre-production stage. A smart farming service at this stage is important for the farmers, which focuses mainly to avoid overproduction or shortage of products that may lead to financial difficulties. 
Scholars faced many difficulties to actualize such ICT onvergence services in the agricultural field to cope with various obstacles such as weather changes, growth condition of farm products and continual disease or technical problems. The proposed system includes service components for planning at this stage. This service needs to be explored more and requires standardization and to contribute to the global usages of smart farming technologies with increase in profit and productivities. The five V's of big data which are the most important, Volume: Large amounts of data, from datasets with sizes of terabytes to zettabyte. Velocity: Large amounts of data from transactions with high refresh rate resulting in data streams coming at great speed and the time to act on the basis of these data streams will often be very short. Variety: Data come from different data sources. Value: It emphasis the statistical events. Veracity: Explains about the trustworthiness and authenticity.

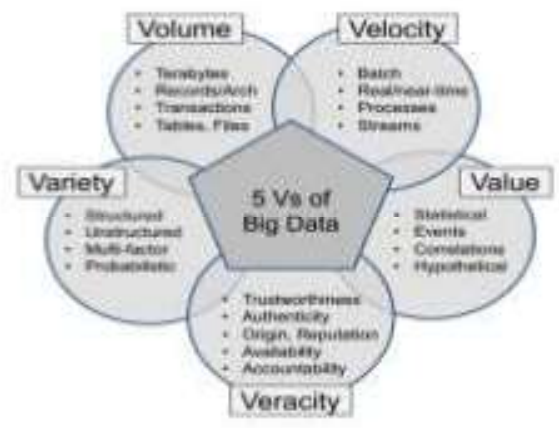

Figure 9: The five V's of Big Data

The above-mentioned technology can be useful for the farmers who are not aware of the current trends. They get latest update about the weather conditions in prior. There are a lot of middle men that play a role in agriculture making it hard for farmers to earn complete profits; the framework mentioned above enables the farmer to have updated information about the current market trends. They are upgraded with web-based learning etc. The tool collects data from various sources and loads the data into HDFS, the queries are examined and processed. Data analysis is done and according to the prediction algorithm the details are given out. The framework is quite affordable and it enables to predict the weather conditions throughout the year. All types of crops can be assessed and grown based on the industry requirement. In one of the article above, the researchers have examined about the new innovations which were developed and furthermore the difficulties confronted focal points and hindrances of the approach proposed. The proposed strategy was to create a framework which could be set up in a little zone by any individual. The upsides of this philosophy were, any individual could execute this framework as it was financially savvy and automatic too and observed the expansion in the product yield. The confronted disservices were to give the required ecological conditions to the plants in order to expand the efficiency. It was observed that the harvest cultivation was accomplished to the level of 5 to 8 times near to the open cultivation. In the research centres, many researches were done using many crops. They influenced utilization of arduino to board fixed with different sensors in them to check the distinctive parameters. The sensors will be initiated by the ecological conditions. The future extension is additionally talked about that is to incorporate remote sensors rather than wired and the arduino board can likewise be supplanted by Raspberry pi as it incorporates in-built database. So, this approach can be enhanced by limiting the expenses by thinking about the fundamental parameters. Another procedure which incorporates different services which helps farmers transcendently. By utilizing these services agriculturists can find out about how to monitor the yields, how one can exceed expectations in advertising the crop, how to oversee fund and numerous more at one stop. The researchers confronted numerous difficulties, preferences and detriments in regards to web services. The usually confronted issue nowadays in the agricultural field is that whether one can take care of the demand for food that is coordinated with step by step developing population. The circumstance of agricultural field has various issues that incorporates less cultivating field, changes in the atmosphere, absence of water, less power supply and the youth search for work elsewhere leaving the matured individuals with cultivating activity. Smart farming is implemented utilizing new innovation with the goal that it turns out to be simple, work minimization and change in crop yielding. The sensors are fixed in the cultivating field so the sensors gather the related data and send it to the server to monitor crops. When the sensors send the informat on to the server, it will naturally activate actions, such as, watering of plants, and so on and different machines have been utilized for traditional works. Focal points were the information which is put away in the server is backed up and particularly secure. The main challenge confronted is the agriculturists must be taught about this innovation, they should be comfortable with utilizing web. The burden is that the cloud innovation requires web 
constantly. If the system is implemented by the farmers the crop yield will be good.

\begin{tabular}{|c|c|c|c|c|c|}
\hline Techaiperer & \multirow{2}{*}{$\begin{array}{l}\text { Seativenany } \\
\text { quiou }\end{array}$} & \multirow{2}{*}{$\begin{array}{c}\text { Pre frublenten } \\
\text { pilas }\end{array}$} & \multirow{2}{*}{ 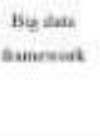 } & \multirow{2}{*}{ 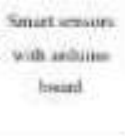 } & \multirow{2}{*}{ 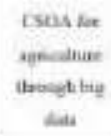 } \\
\hline Criteriz & & & & & \\
\hline Con & Fypesine & Aflatanit & sflachabilt & Aflawitib & Aitsontitis \\
\hline rest & Pavie & Goned & $\begin{array}{l}\text { Ciesed } \\
\text { denumase }\end{array}$ & cond & $\begin{array}{l}\text { Centine } \\
\text { determent }\end{array}$ \\
\hline $\begin{array}{l}\text { Clasi: } \\
\text { endates lin } \\
\text { yuan }\end{array}$ & 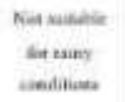 & $\begin{array}{l}\text { Al wriata } \\
\text { centitime }\end{array}$ & 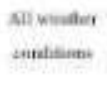 & Nom nants & 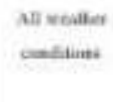 \\
\hline Type af crap & 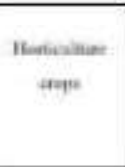 & 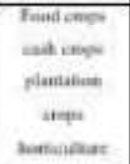 & All vere & Al mpes & 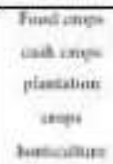 \\
\hline 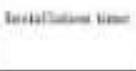 & 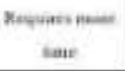 & Lew he & Lesivar: & $\begin{array}{c}\text { Pryuese nuist } \\
\text { Emar }\end{array}$ & Les ue \\
\hline Land Leatnsues & Lappt asole & Lape wate & Larye cenile & Strill acule & Larye acaik \\
\hline
\end{tabular}

\section{CONCLUSION}

Big Data is changing the scope and organization of farming. Global issues such as food security and safety, sustainability and as a result efficiency improvement are tried to be addressed by Big Data applications. These issues make that the scope of Big Data applications extends far beyond farming alone but covers the entire supply chain. Optimization of crops such as planting, watering, pesticide directly affects production rates. Weather predictions and soil moisture sensors allow water wherever needed. Farmers can visualize production levels, soil, sunlight intensity etc. in real time scenario. Automatic processes such as planting and harvesting can reduce consumption, human error and overall cost. Analysing production quality and results can teach farmers to adjust and increase quality of the product. Sensors and machines can be used to detect and manage. Farmers can monitor multiple fields in multiple locations around the globe from an internet connection. Decisions can be made in real-time. Thus, considering the overall cost its usually a onetime investment, it might be a little hard in the initial days but the yield achieved is good. The crops that are grown usually require proper water, manure and pesticides that are looked after by the sensors used. Hence the overall application of big data can improve by the applications of smart farming, though they have lots of drawbacks these can be overcome with time.

\section{References}

[1] Chetan Dwarkani M, Ganesh Ram R, Jagannathan S , R. Priyatharshini “ Smart Farming System Using Sensors for Agricultural Task Automation",2017.

[2] Soong-Hee Lee, Dong-Il Kim, Sok-Pal Cho, Heechang Chung, "Service Model for Smart Farming Services at the Preproduction Stage",2016.

[3] Chandrashekar, Shekar, "Productivity improvement in agriculture sector using big data tools"2015.

[4] Yingli Zhua, Jingjiang Songa, Fuzhou Donga, "Applications of Wireless sensor network in the agriculture environment monitoring" ELSEVIER, Procedia Engineering Sciences-2016.

[5] Shruti A Jaishetty, Rekha Patil, "IoT sensor network-based approach for agricultural field monitoring and control" IJRET: International Journal of Research in Engineering and Technology, 2016.

[6] Baltej Kaur, Danish Inamdar, Vishal Raut, Akash Patil, Nayan Patil, "A Survey On Smart Drip Irrigation System", 2016.

[7] Srisruthi. S, N. Swarna, G. M. Susmitha Ros, Edna Elizabeth, "Sustainable Agriculture using Ecofriendly and Energy Efficient Sensor Technology",2016.

[8] Nikesh Gondchawar, Prof. Dr. R. S. Kawitkar, "IoT based Smart Agriculture", 2016.

[9] R.Ramya.R.Shwetha "Smart Farming Sensors Using Sensors", 2017. 